\title{
Cooperative Output Regulation of Nonlinear Multiagent Systems under a General Directed Graph
}

\author{
Dingcai Huang, Xiangke Wang, Yifeng Niu, and Lincheng Shen \\ College of Mechatronic Engineering and Automation, National University of Defense Technology, Changsha 410073, China \\ Correspondence should be addressed to Xiangke Wang; xkwang@nudt.edu.cn
}

Received 17 April 2015; Accepted 13 September 2015

Academic Editor: Ivanka Stamova

Copyright ( 2015 Dingcai Huang et al. This is an open access article distributed under the Creative Commons Attribution License, which permits unrestricted use, distribution, and reproduction in any medium, provided the original work is properly cited.

\begin{abstract}
This paper studies the cooperative output regulation problem for a class of nonlinear multiagent systems modeled by nonlinear dynamics under a general directed communication topology. A type of distributed internal model is introduced to convert the cooperative output regulation problem into a robust stabilization problem of a so-called augmented system. Based on the Lyapunov stability theorem and $M$-matrix theorem, a kind of distributed output feedback controller only using the relative outputs of neighboring agents together with its stability analysis is further proposed with the aid of the backstepping technology, under which the outputs of the followers will asymptotically converge to that of the leader if, for each follower, there exists at least a directed path from the leader to the follower. Finally, a numerical example is provided to illustrate the effectiveness of the analytic results.
\end{abstract}

\section{Introduction}

In recent decades, the cooperative control problem of distributed dynamic systems has received compelling attentions from various scientific communities for its widespread applications in many areas such as swarm of animals, collection motion of particles, unmanned aerial vehicles, and distributed sensors networks, to name a few. As part of cooperative control, control problems for multiagent systems (MAS) mainly involve designing distributed controllers for each individual agent in the group to make the whole system accomplish specified missions by local interactions with the neighboring agents, due to its lack of global information of the whole system. Several interesting problems in the field have been studied widely, including consensus, formation, flocking, coverage, and leader-following coordination [1-5].

Among these problems, leader-following consensus problem, where the motion of the leader is independent of all other agents and followed by all the other ones, is an active topic recently. The basic idea of the leader-following consensus is that each agent updates its information state on the basis of a consensus protocol. The consensus protocol is an interaction rule that specifies the information exchange between an agent and all of its neighbors in the network [6]. Leader-following consensus algorithms have been investigated in various works [7-11]. Those algorithms take the form of first- or second-order linear dynamics. Meng et al. [11] focus on the leader-following consensus problem for identical linear multiagent systems subject to control input saturation where the linear systems are neutrally stable double integrator systems. In addition, different conditions on communication graphs to achieve the consensus have been explored. Zhu and Cheng [7] give different conditions to guarantee the leader-following consensus of second-order multiagent systems with fixed and switching topologies as well as nonuniform time-varying delays. A common feature of those existing works on leader-following consensus is that the intrinsic nonlinear dynamics of each agent are not considered. In order to investigate and simulate more realistic multiagent systems, the nonlinearity of the agents should be taken into account. Extensions to nonlinear dynamics are studied in $[12,13]$, where the authors discuss the leader-following consensus problems of multiagent systems with nonlinear dynamics. Based on graph theory, matrix theory, and LaSalle's invariance principle, Song et al. [12] propose a pinning control algorithm to solve the 
coupled second-order leader-following consensus problem of nonlinear multiagent systems at a constant reference velocity. However, they assume that the nonlinear term satisfies the Lipschitz condition. For the general nonlinear term with local Lipschitz condition, a consensus algorithm is developed with the aid of the cyclic-small-gain theorem to solve the coordination control problem with second-order dynamics under directed topology in our previous work [5] by using the state feedback of the agents.

It is worth pointing out that most of the existing works consider the state feedback, which requires the states of the agents. In contrast, the output feedback is only using the outputs of the system. In essence, the leader-following consensus problem can be formulated as a cooperative output regulation problem in a uniform framework, due to its strong theoretical and practical background. Cooperative output regulation mainly refers to designing distributed feedback controllers for the considered multiagent systems, such that all the followers can track the active leader and/or reject disturbance signals generated by some so-called exosystem, which has been widely studied in [14-16]. To name a few, $\mathrm{Su}$ and Huang [14] define a general formulation for the cooperative output regulation problem of linear multiagent systems. Wang et al. [15] consider the cooperative output regulation problem of switched linear multiagent systems with saturation input. Li et al. [16] consider a class of heterogeneous multiagent systems with a common reference input but with different disturbances and present two classes of distributed adaptive pinning control laws based on state feedback and dynamic output feedback. Early researches mostly focus on cooperative output regulation problems on the linear multiagent systems and are shifting to the problems on the nonlinear multiagent systems.

Motivated by the above works, this paper investigates the cooperative output regulation problem of multiagent systems with general nonlinear dynamics under a general directed communication topology, where the nonlinear terms are not required to satisfy the Lipschitz condition. In detail, an augmented system is firstly constructed based on the internal model principle, such that the cooperative output regulation problem is transformed into a stabilization problem of the augmented system; then, a distributed control law based on the relative information between neighboring agents under a general directed graph is constructed with the aid of the backstepping design method, which ensures the stability of the original systems. The main contributions focus on the following aspects. (i) The feedbacks used in the control law are the outputs of the systems, rather than the widely used states, which results in wide applications. (ii) The dynamics of agents considered in this paper are with general nonlinear terms, instead of the Lipschitz condition, a rather relaxed assumption which has been used in a wide range of practical nonlinear systems. (iii) The designed consensus protocol only required the relative outputs of the neighboring agents, which means it is distributed, and can easily be scaled up for the larger networked systems. (iv) The communicating topology is directed, and the bidirected graph can be taken as a particular case.
The rest of the paper is organized as follows. Section 2 introduces some notations of the graph theory and gives the problem formulation. In Section 3, a so-called augmented system is constructed based on an internal model, and distributed feedback controllers based on the relative outputs of neighboring agents with their stability analysis are designed by using the backstepping method immediately, which are the main results of this paper. Then, Section 4 presents a simulation example to illustrate our proposed results. Finally, the conclusion is given in Section 5 .

\section{Preliminaries and Problem Formulation}

In this section, preliminary knowledge and problem formulation are introduced.

2.1. Notations and Graph Theory. First of all, we review some preliminary knowledge. The following notation will be used throughout this paper: given the column vectors $a_{i}$, $i=1, \ldots, n$, we denote $\operatorname{col}\left(a_{1}, \ldots, a_{n}\right)=\left[a_{1}^{T}, \ldots, a_{n}^{T}\right]$. Let $I$ be the identity matrix with compatible dimension. For a given matrix $A, A^{T}$ denotes its transpose, and $\lambda_{\max }(A)$ and $\lambda_{\min }(A)$ represent the maximum and minimum eigenvalue of matrix $A$, respectively. A matrix is said to be Hurwitz if all its eigenvalues have negative real parts. A matrix is called a nonsingular $M$-matrix if all its off-diagonal entries are nonpositive and all its eigenvalues have positive real parts.

Lemma 1 (see [17]). For a Hurwitz matrix $A$, there exists a positive definite matrix $P$ such that $A^{T} P+P A \leq-I$.

Lemma 2 (see [17]). If $Z$ is a nonsingular M-matrix, there exists a diagonal matrix $D=\operatorname{diag}\left(d_{1}, \ldots, d_{n}\right)$ with $d_{i}>0$, for $i=1, \ldots, n$, such that $\Pi=D Z+Z^{T} D$ is symmetric and positive definite.

Then, we describe the interaction relationship between the agents in multiagent systems by a simple directed graph $\mathscr{G}=(\mathscr{V}, \varepsilon, A)$ which is consisting of a node set $\mathscr{V}=$ $\{1,2, \ldots, n\}$ and an edge set $\varepsilon \subseteq \mathscr{V} \times \mathscr{V}[18] .\left(v_{i}, v_{j}\right) \in \varepsilon$ implies that node $j$ can access the information of node $i$, but not necessarily vice versa. If an edge $\left(v_{i}, v_{j}\right) \in \varepsilon$, then node $i$ is called a neighbor of node $j$. The set of neighbors of node $i$ is defined as $\mathcal{N}_{i}=\{j \in \mathscr{V} \mid(j, i) \in \varepsilon\} . A=\left[a_{i j}\right] \in \mathbb{R}^{n \times n}$ represents a weighed adjacency matrix associated with graph $\mathscr{G}$, where $a_{i j}>0$ if $\left(v_{j}, v_{i}\right) \in \varepsilon$ and $a_{i j}=0$ otherwise. A diagonal matrix $D=\operatorname{diag}\left\{d_{1}, d_{2}, \ldots, d_{n}\right\}$ with diagonal element $d_{i}=\sum_{j=1}^{n} a_{i j}$ is called the degree matrix of graph. Then, the Laplacian matrix of $\mathscr{G}$ is defined as $L=D-A$. A directed graph $\mathscr{G}$ has a directed spanning tree if and only if there exists at least one node that has a directed path to any other node.

For a multiagent system (labeled as $1, \ldots, n$ ) with a leader (labeled as 0 ), the interaction topology is described by graph $\overline{\mathscr{G}}$, which contains graph $\mathscr{G}$, node $v_{0}$, and edges from node $v_{0}$ to other nodes. Let $B=\operatorname{diag}\left\{a_{1}, \ldots, a_{N}\right\}$, where $a_{i}, i=$ $1, \ldots, n$, is the weight of edge $\left(v_{i}, v_{0}\right)$. And denote the matrix $H=L+B$ to describe the connectivity of graph $\overline{\mathscr{G}}$; then, the matrix $H$ has the following property. 
Lemma 3 (see [19]). All the eigenvalues of matrix $H$ have a positive real part, if and only if the directed graph has a directed spanning tree with node 0 as the root.

2.2. Problem Formulation. Consider the multiagent system consisting of $n$ following agents and a leader. The dynamics of the leader can be described as follows:

$$
\begin{aligned}
\dot{v} & =S v, \\
y_{0} & =q(v, w),
\end{aligned}
$$

where $v \in \mathbb{R}^{n_{v}}$ and $y_{0} \in \mathbb{R}$ are the state variable and measured output of the leader, respectively. $S \in \mathbb{R}^{n_{s} \times n_{s}}$ is a constant matrix, and $q(v, w)$ is a smooth function vanishing at the origin.

For $i=1, \ldots, n$, the $i$ th following agent is represented by the dynamic of the following form:

$$
\begin{aligned}
& \dot{x}_{i}=f\left(x_{i}, y_{i}, w\right), \\
& \dot{y}_{i}=g\left(x_{i}, y_{i}, w\right)+b(w) u_{i}, \\
& e_{i}=y_{i}-y_{0},
\end{aligned}
$$

where $x_{i} \in \mathbb{R}^{n_{x}}, u_{i} \in \mathbb{R}$, and $y_{i} \in \mathbb{R}$ are, respectively, the state variable, control input, and measured output of agent $i . w \in$ $\mathbb{R}^{n_{w}}$ is an uncertain vector and $b(w)>0$. Here, $f\left(x_{i}, y_{i}, w\right)$ and $g\left(x_{i}, y_{i}, w\right)$ are smooth functions vanishing at the origin. Denote $e=\operatorname{col}\left(e_{1}, \ldots, e_{n}\right)$.

Remark 4. According to the definition of the output regulation theory, the control objective is to stabilize the closedloop system consisting of the leader subsystem and the $n$ follower subsystems, such that the regulated errors $e_{1}, \ldots, e_{n}$ converge to zero. For $i=1, \ldots, n$, if $e_{i}$ is available to the $i$ th agent, the cooperative output regulation can be achieved by decentralized output regulation. However, only a part of the follower agents can obtain the information of the leader due to the communication, whereas the others cannot. Therefore, $e_{i}$ cannot be used directly in the controller design. Thus, in general, a so-called virtual regulated error $e_{v i}$ is introduced, which is defined as follows:

$$
e_{v i}=\sum_{j \in \mathcal{N}_{i}} a_{i j}\left(y_{j}-y_{i}\right)+a_{i}\left(y_{i}-y_{0}\right), \quad i=1, \ldots, n .
$$

Denote $e_{v}=\operatorname{col}\left(e_{v 1}, \ldots, e_{v n}\right)$.

In this paper, the distributed dynamic feedback controllers based on the virtual regulated error $e_{i v}$ for agent $i$ are given in the following form:

$$
\begin{aligned}
& u_{i}=k_{i}\left(\eta_{i}, e_{i v}\right), \\
& \dot{\eta}_{i}=h_{i}\left(\eta_{i}, e_{i v}\right),
\end{aligned}
$$

$$
i=1, \ldots, n,
$$

where $\eta_{i}$ is the compensator state vector with compatible dimension to be specified later and $k_{i}$ and $h_{i}$ are some smooth functions vanishing at the origin.
Having defined the notations above, the cooperative output regulation problem of nonlinear multiagent systems can be stated as follows.

Definition 5. The cooperative output regulation problem of systems (1) and (2) with the corresponding communication graph $\overline{\mathscr{G}}$ is achieved with the distributed feedback controller of form (4) if, for any initial states $\left(x_{1}(0), \ldots, x_{N}(0)\right)$, the following two properties are satisfied.

Property 1. The trajectories of the closed-loop composite systems (2) and (4) exist and are bounded, for all $t \geq 0$.

Property 2. For any initial condition $v(0)$, the regulated error $e_{i}$ satisfies

$$
\lim _{t \rightarrow \infty} e_{i}(t)=0, \quad i=1, \ldots, n
$$

Then, we will list some assumptions and definitions for the problem as follows.

Assumption 6. Matrix $S$ has all the eigenvalues with a negative real part.

Assumption 7. There exist smooth functions $\mathbf{x}_{i}(\nu, w), \mathbf{y}_{i}(\nu, w)$, and $\mathbf{u}_{i}(v, w)$ vanishing at the origin, such that, for arbitrary $v \in \mathbb{R}^{n_{v}}$ and $w \in \mathbb{R}^{n_{w}}$,

$$
\begin{aligned}
\frac{\partial \mathbf{x}_{i}(v, w)}{\partial v} S= & f\left(\mathbf{x}_{i}(v, w), \mathbf{y}_{i}(v, w), v, w\right) \\
\frac{\partial \mathbf{y}_{i}(v, w)}{\partial v} S= & g\left(\mathbf{x}_{i}(v, w), \mathbf{y}_{i}(v, w), v, w\right) \\
& +b(w) \mathbf{u}_{i}(v, w) \\
0= & \mathbf{y}_{i}(v, w)-q(v) .
\end{aligned}
$$

Assumption 8 . The solutions $\mathbf{u}_{i}(v, w), i=1, \ldots, n$, are polynomials in $v$ with their coefficients depending on $w$.

Remark 9. According to Theorem 3.8 in [20], Assumption 6 implies that the leader subsystem is neutrally stable. Equations (6) are called regulator equations (REs), and Assumption 7 ensures the solvability of regulator equations, since their solvability is a necessary condition for that of the cooperative output regulation problem. Under Assumption 7, let $\mathbf{y}_{i}(v, w)=q(v, w), u_{i}(v, w)=b_{i}^{-1}(w)((\partial q(v, w) /$ $\left.\partial v) S v-g_{i}\left(\mathbf{z}_{i}(v, w), q(v, w), v, w\right)\right)$; then, it can be verified that $\mathbf{x}_{i}(v, w), \mathbf{y}_{i}(v, w)$, and $\mathbf{u}_{i}(v, w)$ are solutions of the regulator equations. Assumption 8 guarantees the existence of certain linear dynamic equation which is independent of $w$ and can produce the solution or part of the solution of the regulator equations. [21].

Next, we will introduce some concepts of internal model

Definition 10 (steady-state generator). The multiagent systems (1) and (2) are said to have a steady-state generator with output $\mathbf{u}_{i}(\nu, w)$, if there exists a triple $\left\{\theta_{i}, \alpha_{i}, \beta_{i}\right\}$ of smooth 
functions all vanishing at the origin for $i=1, \ldots, n$, such that, for all the trajectories $v(t)$ of the leader subsystem,

$$
\begin{gathered}
\frac{d \theta_{i}(v, w)}{d t}=\alpha_{i}\left(\theta_{i}(v, w)\right), \\
\mathbf{u}_{i}(v, w)=\beta_{i}\left(\theta_{i}(v, w)\right) .
\end{gathered}
$$

In addition, if the pair $\left(\beta_{i}, \alpha_{i}\right)$ is observable, then $\left\{\theta_{i}, \alpha_{i}, \beta_{i}\right\}$ is called a linearly observable steady-state generator with output $\Sigma\left(\mathbf{x}_{i}(v, w), \mathbf{u}_{i}(v, w)\right)$.

Definition 11 (internal model). Under Assumptions 6 and 7, suppose the multiagent systems (1) and (2) have a steady-state generator with output $\mathbf{u}_{i}(v, w)$. Then, the following dynamic system,

$$
\dot{\eta}_{i}=M_{i} \eta_{i}+N_{i} u_{i}, \quad i=1, \ldots, n
$$

is called an internal model with output $\mathbf{u}_{i}(v, w)$, if

$$
M_{i} \theta_{i}+N_{i} \mathbf{u}_{i}(v, w)=\alpha_{i}\left(\theta_{i}(v, w)\right)
$$

\section{The Main Results}

In this section, we will construct the distributed dynamic feedback controller based on the distributed internal model. It is well known that there is a general framework established in Huang and Chen [21] to handle the general nonlinear output regulation. By this methodology, the problem can be fixed in two steps. First, seek a suitable internal model, and the problem can be converted into a robust stabilization problem of a so-called augmented system by a suitable coordinate and input transformation. Second, the robust stabilization problem can be solved by many complex nonlinear design methodologies. Therefore, the controller of the cooperative output regulation problem is composed of two coupled parts: a distributed internal model and a stabilizer.

3.1. Distributed Internal Models. According to the concept of the internal model defined in Definition 11, perform the following coordinate and input transformation:

$$
\begin{aligned}
& \bar{x}_{i}=x_{i}-\mathbf{x}_{i}(v, w), \\
& \tilde{\eta}_{i}=\eta_{i}-\theta_{i}(v, w)-b^{-1}(w) N_{i} e_{i}, \\
& \bar{u}_{i}=u_{i}-\Gamma_{i} T_{i}^{-1} \eta_{i} .
\end{aligned}
$$

Then, the following augmented system (11) is obtained:

$$
\begin{aligned}
& \dot{\bar{x}}_{i}=\bar{f}\left(\bar{x}_{i}, e_{i}, \mu\right), \\
& \dot{\tilde{\eta}}_{i}=M_{i} \widetilde{\eta}_{i}+\bar{\varphi}\left(\bar{x}_{i}, e_{i}, \mu\right), \\
& \dot{e}_{i}=\bar{g}\left(\bar{x}_{i}, \widetilde{\eta}_{i}, e_{i}, \mu\right)+b(w) \bar{u}_{i},
\end{aligned}
$$

where $\mu(t)=\operatorname{col}(v(t), w)$ and

$$
\begin{aligned}
& \bar{f}_{i}\left(\bar{x}_{i}, e_{i}, \mu\right)= f\left(\bar{x}_{i}+\mathbf{x}_{i}, e_{i}+q(v, w), v, w\right) \\
&-f\left(\mathbf{x}_{i}, q(v, w), v, w\right), \\
& \bar{\varphi}_{i}\left(\bar{x}_{i}, e_{i}, \mu\right)=b^{-1}(w) M_{i} N_{i} e_{i} \\
&-b^{-1}(w) N_{i} \omega_{i}\left(\bar{x}_{i}, e_{i}, \mu\right), \\
& \bar{g}_{i}\left(\bar{x}_{i}, \tilde{\eta}_{i}, e_{i}, \mu\right)=\omega_{i}\left(\bar{x}_{i}, e_{i}, \mu\right)+b(w) \Psi_{i} \tilde{\eta}_{i}+\Psi_{i} N_{i} e_{i}, \\
& \bar{w}_{i}\left(\bar{x}_{i}, e_{i}, \mu\right)=g\left(\bar{x}_{i}+\mathbf{x}_{i}(v, w), e_{i}+q(v, w), v, w\right) \\
&-f\left(\mathbf{x}_{i}(v, w), q(v, w), v, w\right) .
\end{aligned}
$$

Now we can start to construct a suitable distributed internal model for the system. The following theorem shows the existence of a distributed linear internal model for the multiagent systems and how to solve the cooperative output regulation problem on the basis of the internal model.

Theorem 12. Under Assumptions 6-8, if there exist distributed feedback controllers $\bar{u}_{i}=k_{i}\left(e_{v i}\right)$ stabilizing augmented system (11), then there exist controllable pairs $\left(M_{i}, N_{i}\right), M_{i} \in \mathbb{R}^{n_{i} \times n_{i}}$ is a Hurwitz matrix, and $N_{i} \in \mathbb{R}^{n_{i} \times 1}$ is a column vector, such that the cooperative output regulation problem of multiagent systems (1) and (2) can be solved by the following controllers:

$$
\begin{aligned}
& u_{i}=k_{i}\left(e_{v i}\right)+\Gamma_{i} T_{i}^{-1} \eta_{i}, \\
& \dot{\eta}_{i}=M_{i} \eta_{i}+N_{i} u_{i},
\end{aligned}
$$

$$
i=1, \ldots, n,
$$

where a nonsingular matrix $T_{i}$ satisfies the Sylvester equation,

$$
T_{i} \Phi_{i}-M_{i} T_{i}=N_{i} \Gamma_{i}
$$

and $\Gamma_{i}$ is a column vector.

Proof. Under Assumption 8, there exist integers $n_{i}(i=$ $1, \ldots, n)$, such that, for all $w \in \mathbb{W}, \mathbf{u}_{i}(v, w)$ satisfies [20]:

$$
\begin{aligned}
\frac{d^{n_{i}} \mathbf{u}_{i}(v, w)}{d t^{n_{i}}}= & a_{1 i} \mathbf{u}_{i}(v, w)+a_{2 i} \frac{d \mathbf{u}_{i}(v, w)}{d t}+\cdots \\
& +a_{n_{i} i} \frac{d^{\left(n_{i}-1\right)} \mathbf{u}_{i}(v, w)}{d t^{\left(n_{i}-1\right)}} .
\end{aligned}
$$

Then, denote

$$
\begin{aligned}
\Phi_{i} & =\left[\begin{array}{c|c}
0 & I_{n_{i}-1} \\
\hline a_{1 i} & a_{2 i}, \ldots, a_{n_{i} i}
\end{array}\right]_{n_{i} \times n_{i}}, \\
\Gamma_{i} & =\left[\begin{array}{llll}
1 & 0 & \cdots & 0
\end{array}\right]_{1 \times n_{i}} .
\end{aligned}
$$

It can be verified that $\left(\Gamma_{i}, \Phi_{i}\right)$ is observable. Then, it is noted that the spectra of $\Phi_{i}$ and $M_{i}$ are disjoint; therefore, there exists a nonsingular matrix $T_{i}$ satisfying the Sylvester equation:

$$
T_{i} \Phi_{i}-M_{i} T_{i}=N_{i} \Gamma_{i}
$$


Consequently, define $\theta_{i}(v, w)=T_{i} \operatorname{col}\left(\mathbf{u}_{i}(v, w), d \mathbf{u}_{i}(v\right.$, $\left.w) / d t, \ldots, d^{\left(n_{i}-1\right)} \mathbf{u}_{i}(v, w) / d t^{\left(n_{i}-1\right)}\right)$. Then, it can be verified that

$$
\begin{gathered}
\frac{d \theta_{i}(v, w)}{d t}=T_{i} \Phi_{i} T_{i}^{-1} \theta_{i}(v, w), \\
\mathbf{u}_{i}(v, w)=\Gamma_{i} T_{i}^{-1} \theta_{i}(v, w) .
\end{gathered}
$$

Thus, by Definition 10, the triple $\left\{\theta_{i}(v, w), T_{i} \Phi_{i} T_{i}^{-1} \theta_{i}(v\right.$, $\left.w), \Gamma_{i} T_{i}^{-1} \theta_{i}(v, w)\right\}$ is a steady-state generator with output $\mathbf{u}_{i}(v, w)$ for the multiagent system.

Then, it can be verified that

$$
\begin{aligned}
M_{i} & \theta_{i}(v, w)+N_{i} \mathbf{u}_{i}(v, w) \\
& =M_{i} \theta_{i}(v, w)+N_{i} \Gamma_{i} T_{i}^{-1} \theta_{i}(v, w) \\
& =\left(M_{i}+N_{i} \Gamma_{i} T_{i}^{-1}\right) \theta_{i}(v, w)=T_{i} \Phi_{i} T_{i}^{-1} \theta_{i}(v, w) .
\end{aligned}
$$

Therefore, according to Definition $11, \dot{\eta}_{i}=M_{i} \eta_{i}+N_{i} u_{i}$ is an internal model for each follower with output $\mathbf{u}_{i}(v, w)$.

According to Corollary 6.9 in Huang [20], it is known that if a controller solves the stabilization problem for the augmented system, then this controller together with the internal model solves the output regulation for the original system. Therefore, due to the distributed feedback controllers $\bar{u}_{i}=k_{i}\left(e_{v i}\right)$ stabilizing augmented system (11), controllers (13) solve the cooperative output regulation problem of multiagent systems (1) and (2).

Thus, the proof is completed.

Remark 13. Note that there are a variety of internal models with output $\mathbf{u}_{i}(v, w)$. In fact, the steady-state generator itself is an internal model. The reason for choosing the internal model presented above is that the Hurwitz property of matrix $M_{i}$ can lead to an augmented system whose robust stabilization problem is solvable, and it has advantages in the stabilization design shown later in the next subsection; however, the steady-state generator does not.

In addition, it is noted that an equilibrium of augmented system (11) is at $\left(\bar{x}_{i}, \eta_{i}, e_{i}\right)$, for all $v \in \mathbb{V}$. As long as a stabilizer $\bar{u}_{i}$ can be found such that the augmented system is globally asymptotically stable at the equilibrium, the cooperative output regulation problem will be solved.

3.2. Distributed Stabilization of Augmented System. Here, we will adopt the backstepping method to construct a suitable stabilizer for the augmented system, only by using relative information of the outputs between neighboring agents. Firstly, an important property of continuous function is given as follows.

It is known that since $\bar{\varphi}_{i}\left(\bar{x}_{i}, e_{i}, \mu\right)$ is a smooth function vanishing at the origin, then there exist some real constant $c_{i}$ and some smooth positive functions $\pi_{1 i}\left(\bar{x}_{i}\right)$ and $\pi_{2 i}\left(e_{i}\right)$, such that, for all $\mu \in \Sigma$,

$$
\left\|\bar{\varphi}_{i}\left(\bar{x}_{i}, e_{i}, \mu\right)\right\| \leq c_{i}\left[\pi_{1 i}\left(\bar{x}_{i}\right)\left\|\bar{x}_{i}\right\|+\pi_{2 i}\left(e_{i}\right)\left\|e_{i}\right\|\right] .
$$

Denote $X_{i}=\operatorname{col}\left(\bar{x}_{i}, \widetilde{\eta}_{i}\right)$; then, it is the same with the function $\bar{g}_{i}\left(X_{i}, e_{i}\right)$. It is known that there exist some real constant $\varepsilon_{i}$ and some smooth positive functions $\pi_{3 i}\left(X_{i}\right)$ and $\pi_{4 i}\left(e_{i}\right)$, such that, for all $\mu \in \Sigma$,

$$
\left\|\bar{g}_{i}\left(X_{i}, e_{i}\right)\right\| \leq \varepsilon_{i}\left[\pi_{3 i}\left(X_{i}\right)\left\|X_{i}\right\|^{2}+\pi_{4 i}\left(e_{i}\right) e_{i}^{2}\right] .
$$

Now, the main results are given as follows.

Theorem 14. Suppose $\overline{\mathscr{G}}$ is a directed graph which contains a directed spanning tree with node 0 as the root. Then, under Assumptions 6-8, there exists a controller of the following form:

$$
\begin{aligned}
\bar{u}_{i} & =-\frac{1+(1 / 2) \xi_{\max } \lambda_{\max }\left(H^{T} H\right)\left(\varepsilon_{i}+\max \pi_{4 i}\left(e_{i}\right)\right)}{b(w) \lambda_{\min }(R) \lambda_{\min }\left(H^{T} H\right)} \\
& \cdot e_{v i}, \quad i=1, \ldots, n,
\end{aligned}
$$

such that augmented system (11) is globally asymptotically stable.

Proof. Firstly, denote $k_{i}\left(e_{v i}\right)=\left(1+(1 / 2) \xi_{\max } \lambda_{\max }\left(H^{T} H\right)\left(\varepsilon_{i}+\right.\right.$ $\left.\left.\max \pi_{4 i}\left(e_{i}\right)\right)\right) / b(w) \lambda_{\min }(R) \lambda_{\min }\left(H^{T} H\right)$. Note that $k_{i}(\cdot)$ is a positive smooth function. We will adopt the backstepping method as follows.

Step 1. Consider the $\bar{x}_{i}$-subsystem of (11) and treat the variable $e_{i}$ as a virtual control input. Assume that there exist a feedback control $e_{i}=\phi_{i}\left(\bar{x}_{i}\right) \bar{x}_{i}$ stabilizing the $\bar{x}_{i}$-subsystem and a smooth and positive definite Lyapunov function candidate $V_{1 i}$ satisfying

$$
\dot{V}_{1 i}=\frac{\partial V_{1}}{\partial \bar{x}_{i}} \bar{f}_{i} \leq-\Delta_{i}\left(\bar{x}_{i}\right)\left\|\bar{x}_{i}\right\|^{2} .
$$

Step 2. Consider the $\left(\bar{x}_{i}, \widetilde{\eta}_{i}\right)$-subsystem and treat the variable $e_{i}$ as a virtual control input. Since $M_{i}$ is Hurwitz, there exists a nonsingular matrix $P_{i}$ satisfying $M_{i}^{T} P_{i}+P_{i} M_{i} \leq-I$.

Now choose a smooth and positive definite function $V_{2 i}\left(\bar{x}_{i}, \widetilde{\eta}_{i}\right)=l_{i} V_{1 i}\left(\bar{x}_{i}\right)+2 \widetilde{\eta}_{i}^{T} P_{i} \widetilde{\eta}_{i}$ as a Lyapunov function candidate. Then, the derivation of $V_{2 i}$ along the trajectory of $\left(\bar{x}_{i}, \widetilde{\eta}_{i}\right)$-subsystem is given by the following:

$$
\begin{aligned}
\dot{V}_{2 i} & =l_{i} \dot{V}_{1 i}\left(\bar{x}_{i}\right)+2 \widetilde{\eta}_{i}^{T}\left(M_{i}^{T} P_{i}+P_{i} M_{i}\right) \widetilde{\eta}_{i} \\
& +4 \bar{\varphi}_{i}^{T}\left(\bar{x}_{i}, e_{i}, \mu\right) P_{i} \widetilde{\eta}_{i} \leq-l_{i} \Delta_{i}\left(\bar{x}_{i}\right)\left\|\bar{x}_{i}\right\|^{2}-2\left\|\widetilde{\eta}_{i}\right\|^{2} \\
& +4\left\|P_{i}\right\|^{2} \bar{\varphi}_{i}^{2}\left(\bar{x}_{i}, e_{i}, \mu\right)+\widetilde{\eta}_{i}^{2} \leq-l_{i} \Delta_{i}\left(\bar{x}_{i}\right)\left\|\bar{x}_{i}\right\|^{2} \\
& -\left\|\tilde{\eta}_{i}\right\|^{2}+4 c_{i}^{2}\left\|P_{i}\right\|^{2}\left[\pi_{1 i}^{2}\left(\bar{x}_{i}\right)\left\|\bar{x}_{i}\right\|^{2}+\pi_{2 i}^{2}\left(e_{i}\right) e_{i}^{2}\right] \\
& \leq-\left\{l_{i} \Delta_{i}\left(\bar{x}_{i}\right)\right. \\
& \left.-4 c_{i}^{2}\left\|P_{i}\right\|^{2}\left[\pi_{1 i}^{2}\left(\bar{x}_{i}\right)+\pi_{2 i}^{2}\left(\phi_{i}\left(\bar{x}_{i}\right)\right) \phi_{i}^{2}\left(\bar{x}_{i}\right)\right]\right\}\left\|\bar{x}_{i}\right\|^{2} \\
& -\left\|\tilde{\eta}_{i}\right\|^{2} .
\end{aligned}
$$

If we choose $\Delta_{i}\left(\bar{x}_{i}\right) \geq 1+\pi_{1}^{2}\left(\bar{x}_{i}\right)+\pi_{2}^{2}\left(\phi_{i}\left(\bar{x}_{i}\right)\right) \phi_{i}^{2}\left(\bar{x}_{i}\right)$ and $l_{i}=$ $\max \left\{1,4 c_{i}^{2}\left\|P_{i}\right\|^{2}\right\}$, then

$$
\dot{V}_{2 i} \leq-\left\|\bar{x}_{i}\right\|^{2}-\left\|\widetilde{\eta}_{i}\right\|^{2} .
$$


Then, denote $X=\operatorname{col}\left(X_{1}, \ldots, X_{n}\right)$. Consider the $X$ subsystem, and let $V_{2}(X)=\sum_{i=1}^{n} V_{2 i}\left(X_{i}\right)$. Then, the derivation of $V(X)$ along the trajectory of $X$-subsystem is given by the following:

$$
\begin{aligned}
\dot{V}_{2}(X) & =\sum_{i=1}^{n} \dot{V}_{2 i}\left(X_{i}\right) \leq \sum_{i=1}^{n}\left(-\left\|\bar{x}_{i}\right\|^{2}-\left\|\tilde{\eta}_{i}\right\|^{2}\right) \\
& \leq-\sum_{i=1}^{n}\left\|X_{i}\right\|^{2} \leq-\|X\|^{2} .
\end{aligned}
$$

Step 3. Consider the whole augmented system (11) and treat the variable $\bar{u}_{i}$ as a control input. Since $\overline{\mathscr{G}}$ contains a directed spanning tree with node 0 as the root, by Lemma 3 , all the eigenvalues of $H$ have a positive real part; that is, $H$ is a nonsingular $M$-matrix. Then, by Lemma 2, there exists a positive definite matrix $Q=\operatorname{diag}\left\{\xi_{1}, \ldots, \xi_{n}\right\}$, such that the matrix $R=H^{T} Q+Q H$ is positive definite. Now, let $V(X, e)=$ $r V_{2}(X)+(1 / 2) e_{v}^{T} Q e_{v}$. Then, differentiating $V(X, e)$ along the trajectory of system (11) yields

$$
\begin{aligned}
& \dot{V}(X, e)=r \dot{V}_{2}+e_{v}^{T} Q \dot{e}_{v}=r \dot{V}_{2}+\sum_{i=1}^{n} \xi_{i} e_{v i}^{T} \dot{e}_{v i} \\
& =r \dot{V}_{2}+\sum_{i=1}^{n} \xi_{i} e_{v i}^{T} \sum_{j=0}^{n} a_{i j}\left(\dot{e}_{i}-\dot{e}_{j}\right) \\
& =r \dot{V}_{2} \\
& \quad+\sum_{i=1}^{n} \xi_{i} e_{v i}^{T} \sum_{j=0}^{n} a_{i j}\left(\bar{g}_{i}+b(w) \bar{u}_{i}-\bar{g}_{j}-b(w) \bar{u}_{j}\right) \\
& =r \dot{V}_{2}+\sum_{i=1}^{n} \xi_{i} e_{v i}^{T} \sum_{j=0}^{n} a_{i j}\left(\bar{g}_{i}-\bar{g}_{j}\right) \\
& \quad+b(w) \sum_{i=1}^{n} \xi_{i} e_{v i}^{T} \sum_{j=0}^{n} a_{i j}\left(\bar{u}_{i}-\bar{u}_{j}\right) .
\end{aligned}
$$

Now, define $\gamma_{i}=\sum_{j=0}^{n} a_{i j}\left(\bar{g}_{i}-\bar{g}_{j}\right)$, and denote $\bar{g}=$ $\operatorname{col}\left(\bar{g}_{1}, \ldots, \bar{g}_{N}\right), \bar{u}=\operatorname{col}\left(\bar{u}_{1}, \ldots, \bar{u}_{N}\right) \gamma=\operatorname{col}\left(\gamma_{1}, \ldots, \gamma_{N}\right)=$ $H \bar{g}$, and then we can get $\gamma^{2}=\bar{g}^{T} H^{T} H \bar{g} \leq \lambda_{\max }\left(H^{T} H\right) \bar{g}^{T} \bar{g}$. Moreover, let $K\left(e_{v}\right)=\operatorname{diag}\left(k_{i}\left(e_{v i}\right)\right)$. Therefore,

$$
\begin{aligned}
\dot{V}= & r \dot{V}_{2}+\sum_{i=1}^{n} \xi_{i} e_{v i}^{T} \sum_{j=0}^{n} a_{i j}\left(\bar{g}_{i}-\bar{g}_{j}\right) \\
& +\sum_{i=1}^{n} \xi_{i} e_{v i}^{T} \sum_{j=0}^{n} a_{i j}\left(b(w) \bar{u}_{i}-b(w) \bar{u}_{j}\right) \\
\leq & r \dot{V}_{2}+\sum_{i=1}^{n} \frac{\varepsilon_{i}}{2} \xi_{i} e_{v i}^{2}+\sum_{i=1}^{n} \frac{1}{2 \varepsilon_{i}} \xi_{i} \gamma_{i}^{2}+b(w) e_{v}^{T} Q H \bar{u} \\
\leq & -r\|X\|^{2}+\frac{\varepsilon_{i}}{2} \xi_{\max } e_{v}^{2}+\frac{1}{2 \varepsilon_{i}} \xi_{\max } \lambda_{\max }\left(H^{T} H\right) \bar{g}^{2} \\
& -b(w) e_{v}^{T} Q H K\left(e_{v}\right) e_{v}
\end{aligned}
$$

$$
\begin{aligned}
\leq & -r\|X\|^{2}+\frac{\varepsilon_{i}}{2} \xi_{\max } e_{v}^{2}+\frac{1}{2 \varepsilon_{i}} \xi_{\max } \lambda_{\max }\left(H^{T} H\right) \sum_{i=1}^{n} \bar{g}_{i}^{2} \\
& -b(w) k_{\min } e_{v}^{T} Q H e_{v} \\
\leq & -r\|X\|^{2}+\frac{\varepsilon_{i}}{2} \xi_{\max } e_{v}^{2}+\frac{1}{2 \varepsilon_{i}} \xi_{\max } \lambda_{\max }\left(H^{T} H\right) \\
& -\frac{1}{2} b(w) k_{\min } e_{v}^{T}\left(Q H+H^{T} Q\right) e_{v} \\
\leq & -r\|X\|^{2}+\frac{\varepsilon_{i}}{2} \xi_{\max } e_{v}^{2}+\frac{1}{2 \varepsilon_{i}} \xi_{\max } \lambda_{\max }\left(H^{T} H\right) \sum_{i=1}^{n} \bar{g}_{i}^{2} \\
& -b(w) k_{\min } e_{v}^{T} R e_{v} \\
\leq & -r \sum_{i=1}^{n}\left\|X_{i}\right\|^{2}+\frac{\varepsilon_{i}}{2} \xi_{\max } e_{v}^{2} \\
& +\frac{1}{2 \varepsilon_{i}} \xi_{\max } \lambda_{\max }\left(H^{T} H\right) \sum_{i=1}^{n} \bar{g}_{i}^{2} \\
& -b(w) k_{\min } \lambda_{\min }(R) e_{v}^{2} .
\end{aligned}
$$

Since $e_{v}=H e$, then $\lambda_{\text {min }}\left(H^{T} H\right) e^{2} \leq e_{v} \leq \lambda_{\max }\left(H^{T} H\right) e^{2}$. According to (21), we can obtain the following:

$$
\begin{aligned}
\dot{V} \leq & -r \sum_{i=1}^{n}\left\|X_{i}\right\|^{2}+\frac{\varepsilon_{i}}{2} \xi_{\max } e_{v}^{2}+\frac{1}{2 \varepsilon_{i}} \xi_{\max } \lambda_{\max }\left(H^{T} H\right) \\
& \cdot \sum_{i=1}^{n} \varepsilon_{i}\left[\pi_{3 i}\left(X_{i}\right)\left\|X_{i}\right\|^{2}+\pi_{4 i}\left(e_{i}\right) e_{i}^{2}\right]-b(w) \\
& \cdot k_{\min }\left(e_{v i}\right) \lambda_{\min }(R) e_{v}^{2} \leq-\sum_{i=1}^{n}(r \\
& \left.-\frac{1}{2} \xi_{\max } \lambda_{\max }\left(H^{T} H\right) \pi_{3 i}\left(X_{i}\right)\right)\left\|X_{i}\right\|^{2} \\
& -\sum_{i=1}^{n}\left(-\frac{1}{2} \xi_{\max } \lambda_{\max }\left(H^{T} H\right)\left(\varepsilon_{i}+\pi_{4 i}\left(e_{i}\right)\right)\right. \\
& \left.+b(w) k_{\min }\left(e_{v i}\right) \lambda_{\min }(R) \lambda_{\min }\left(H^{T} H\right)\right) e_{i}^{2} .
\end{aligned}
$$

Choose $r \geq\left(1 / 2 \varepsilon_{i}\right) \xi_{\max } \lambda_{\max }\left(H^{T} H\right) \pi_{3 i}\left(z_{i}\right)+1, k_{i}\left(e_{v i}\right)=$ $\left(1+(1 / 2) \xi_{\max } \lambda_{\max }\left(H^{T} H\right)\left(\varepsilon_{i}+\max \pi_{4 i}\left(e_{i}\right)\right)\right) /$ $b(w) \lambda_{\text {min }}(R) \lambda_{\text {min }}\left(H^{T} H\right)$; then, finally we can obtain the following:

$$
\left.\dot{V}\right|_{26} \leq-\sum_{i=1}^{n}\left\|X_{i}\right\|^{2}-\sum_{i=1}^{n} e_{i}^{2}=-\|X\|^{2}-e^{2} .
$$

The proof is thus completed.

Then, by Theorems 12 and 14, we can give our results of the following theorem. 
Theorem 15. Suppose $\overline{\mathscr{G}}$ is a directed graph which contains a directed spanning tree with node 0 as the root. Then, under Assumptions 6-8, the cooperative output regulation problem of system (2) can be solved by the following:

$$
\begin{aligned}
u_{i} & \\
=- & \frac{1+(1 / 2) \xi_{\max } \lambda_{\max }\left(H^{T} H\right)\left(\varepsilon_{i}+\max \pi_{4 i}\left(e_{i}\right)\right)}{b(w) \lambda_{\min }(R) \lambda_{\min }\left(H^{T} H\right)} e_{v i} \\
& +\Gamma_{i} T_{i}^{-1} \eta_{i}, \\
\dot{\eta}_{i}= & M_{i} \eta_{i}+N_{i} u_{i},
\end{aligned}
$$

$$
i=1, \ldots, N \text {. }
$$

Proof. According to Theorem 14, the augmented system can be stabilized by controller (22). Further, according to Theorem 12, the cooperative output regulation problem can be solved by controller (31). This proof is thus concluded.

Remark 16. A special case is that the communication topology among the followers is bidirected. In this case, we can get $H e=H^{T} e=e_{v}$; then, controller (31) can be rewritten as follows:

$$
\begin{aligned}
u_{i}= & -\frac{1+(1 / 2) \xi_{\max } \lambda_{\max }\left(H^{2}\right)\left(\varepsilon_{i}+\max \pi_{4 i}\left(e_{i}\right)\right)}{b(w) \lambda_{\min }(R) \lambda_{\min }\left(H^{2}\right)} e_{v i} \\
& +\Gamma_{i} T_{i}^{-1} \eta_{i}, \\
\dot{\eta}_{i}= & M_{i} \eta_{i}+N_{i} u_{i},
\end{aligned}
$$

$$
i=1, \ldots, N \text {, }
$$

which can also solve the cooperative output regulation problem of system (2).

\section{Simulation Results}

In this section, numerical simulations are given to illustrate our design. In our simulation, the time step is fixed for 0.001 , and the initial values are randomly taken from -8 , 8 . In particular, consider a group of five followers described as van der Pol oscillators [22]:

$$
\begin{aligned}
\dot{x}_{1 i} & =x_{2 i}, \\
\dot{x}_{2 i} & =-a_{1 i} x_{1 i}+a_{2 i} x_{2 i}-a_{2 i} x_{1 i}^{2} x_{2 i}+b_{i} u_{i}, \\
y_{i} & =x_{2 i}, \\
e_{i} & =y_{i}-v_{1},
\end{aligned}
$$

$$
i=1, \ldots, 5 \text {, }
$$

where $a_{1 i}>0, a_{2 i}>0$, and $b_{i}>0$ are some constant parameters and $v_{1}$ is generated by the leader system $\dot{v}_{1}=\sigma v_{2}$, $\dot{v}_{2}=-\sigma v_{1}, \sigma=0.8$. Let $a_{i}=\left(a_{1 i}, a_{2 i}, b_{i}\right)^{T}=\bar{a}_{i}+\Delta a_{i}$, where $\bar{a}_{i}$ and $\Delta a_{i}$ denote the nominal value and the perturbed values of $a_{i}$, respectively. Assume that $\bar{a}_{i}=[1,1,1]^{T}$,
$v(0)=[0,2]^{T}, \Delta a_{1}=[0.2,-0.1,0.01]^{T}, \Delta a_{2}=$ $[0.1,0.1,-0.02]^{T}, \Delta a_{3}=[-0.03,-0.05,0]^{T}, \Delta a_{4}=$ $[-0.05,0.08,0.05]^{T}$, and $\Delta a_{5}=[0.02,0.1,-0.06]^{T}$.

We can denote $\mathbf{x}_{i}(\nu, w, \sigma)=-(1 / \sigma) v_{2}$, and then $\mathbf{u}_{i}(\nu, w, \sigma)$ has the following properties:

$$
\begin{aligned}
& \mathbf{u}_{i}(v, w, \sigma)=b_{i}^{-1}\left(-a_{2 i} v_{1}+\left(\sigma-\frac{a_{1 i}}{\sigma}\right) v_{2}-\frac{a_{2 i}}{\sigma} v_{1} v_{2}^{2}\right) \\
& \frac{d \mathbf{u}_{i}(v, w, \sigma)}{d t}=b_{i}^{-1}\left(-a_{2 i} \sigma v_{2}-\left(\sigma^{2}-a_{1 i}\right) v_{1}\right. \\
& \left.\quad+2 a_{2 i} v_{1}^{2} v_{2}-a_{2 i} v_{2}^{3}\right) \\
& \frac{d^{2} \mathbf{u}_{i}(v, w, \sigma)}{d t^{2}}=b_{i}^{-1}\left(a_{2 i} \sigma^{2} v_{1}-\left(\sigma^{2}-a_{1 i}\right) \sigma v_{2}\right. \\
& \left.\quad+7 a_{2 i} \sigma v_{1} v_{2}^{2}-2 a_{2 i} \sigma v_{1}^{3}\right) \\
& \frac{d^{3} \mathbf{u}_{i}(v, w, \sigma)}{d t^{3}}=b_{i}^{-1}\left(a_{2 i} \sigma^{3} v_{2}+\sigma^{2}\left(\sigma^{2}-a_{1 i}\right) v_{1}\right. \\
& \left.\quad-20 a_{2 i} \sigma^{2} v_{1}^{2} v_{2}+7 a_{2 i} \sigma^{2} v_{2}^{3}\right) \\
& \frac{d^{4} \mathbf{u}_{i}(v, w, \sigma)}{d t^{4}}=b_{i}^{-1}\left(-a_{2 i} \sigma^{4} v_{1}+\sigma^{3}\left(\sigma^{2}-a_{1 i}\right) v_{2}\right. \\
& \left.-61 a_{2 i} \sigma^{3} v_{1} v_{2}^{2}+20 a_{2 i} \sigma^{3} v_{1}^{3}\right) .
\end{aligned}
$$

It can be verified that $\mathbf{u}_{i}(v, w, \sigma)$ satisfies that $d^{4} \mathbf{u}_{i}(v, w, \sigma) / d t^{4}=-9 \sigma^{4} \mathbf{u}_{i}(v, w, \sigma)-10 \sigma^{2}\left(d^{2} \mathbf{u}_{i}(v, w, \sigma) / d t^{2}\right)$. Therefore, we can choose that

$$
\begin{gathered}
\Phi_{i}(\sigma)=\left[\begin{array}{rrrr}
0 & 1 & 0 & 0 \\
0 & 0 & 1 & 0 \\
0 & 0 & 0 & 1 \\
-9 \sigma^{4} & 0 & -10 \sigma^{2} & 0
\end{array}\right], \\
\Gamma_{i}=\left[\begin{array}{l}
1 \\
0 \\
\vdots \\
0
\end{array}\right]^{T} .
\end{gathered}
$$

And let

$$
M_{i}=\left[\begin{array}{cccc}
0 & 1 & 0 & 0 \\
0 & 0 & 1 & 0 \\
0 & 0 & 0 & 1 \\
-4 & -12 & -13 & -6
\end{array}\right]
$$

$$
N_{i}=\left[\begin{array}{l}
0 \\
0 \\
0 \\
1
\end{array}\right] .
$$




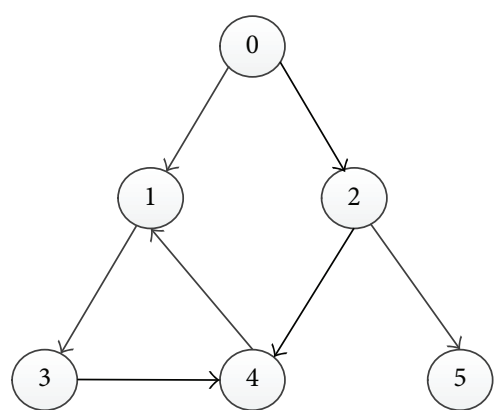

FIGURE 1: The network topology of a multiagent system.

Then, the unique solution of the Sylvester equation (14) is obtained

$$
T_{i}=\left[\begin{array}{cccc}
-0.0749 & 0.1592 & -0.0115 & 0.0285 \\
0.1052 & 0.0749 & 0.0234 & 0.0115 \\
0.0424 & -0.1052 & -0.0013 & -0.0234 \\
-0.0864 & -0.0424 & -0.0449 & 0.0013
\end{array}\right] .
$$

By the coordinate and input transformation, we can get the augmented system:

$$
\begin{aligned}
\bar{z}_{i} & =e_{i}, \\
\dot{\tilde{\eta}} & =M_{i} \widetilde{\eta}+b_{i}^{-1} M_{i} N_{i} e_{i}-b_{i}^{-1} N_{i} \omega\left(\bar{z}_{i}, e_{i}, \mu\right), \\
\dot{e}_{i} & =\omega\left(\bar{z}_{i}, e_{i}, \mu\right)+b_{i} \Psi_{i}^{\sigma} \widetilde{\eta}_{i}+\Psi_{i}^{\sigma} N_{i} e_{i}+b_{i}\left(u_{i}-\Psi_{i}^{\sigma} \eta_{i}\right),
\end{aligned}
$$

where $\omega\left(\bar{z}_{i}, e_{i}, \mu\right)=-a_{1 i} \bar{z}_{i}+a_{2 i} e_{i}-a_{2 i}\left(\bar{z}_{i}+\mathbf{z}_{i}\right)^{2}\left(e_{i}+v_{1}\right)+a_{2 i} z_{i}^{2} v_{1}$.

Figure 1 shows the directed graph $\overline{\mathscr{G}}$ with six agents, labeled from 0 to 5 , where node 0 represents the leader and nodes 1-5 represent the followers. Obviously, the directed graph has a directed spanning tree with node 0 being the root. The corresponding weighted adjacency matrix is described by

$$
H=\left[\begin{array}{ccccc}
2 & 0 & 0 & -1 & 0 \\
0 & 1 & 0 & 0 & 0 \\
-1 & 0 & 1 & 0 & 0 \\
0 & -1 & -1 & 2 & 0 \\
0 & -1 & 0 & 0 & 1
\end{array}\right] .
$$

Then, we can get $\lambda_{\text {min }}\left(H^{T} H\right)=0.2127$ and $\lambda_{\max }\left(H^{T} H\right)=$ 7.8299. Take $Q=\operatorname{diag}(0.1592,0.5308,0.1393,0.0910$, $0.0796)$, and by $H Q+Q H^{T}=R$, we can obtain $\lambda_{\text {min }}(R)=$ 0.1325 . It can be verified that $R$ is positive definite. Thus, we can design the distributed feedback controllers of form (31) by Theorem 15 with $\xi_{\max }=0.5308, \varepsilon=1$, and $\pi_{i}\left(e_{v i}\right)=5\left(e_{v i}^{2}+1\right)$.

In the simulation, the results about the tracking performance for the nonlinear multiagent systems are shown in Figures 2 and 3. Figure 2 shows the outputs of the leader and all the followers, while Figure 3 shows the response of the regulated errors $e_{i}(t)$. It is observed that, with parameter perturbations, the outputs of all the followers asymptotically approach that of the leader, and the regulated errors of all

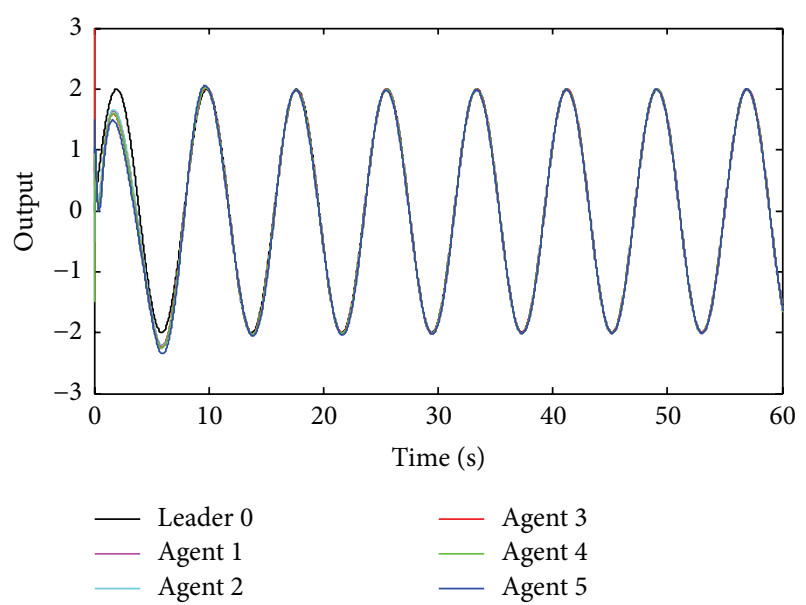

FIgURE 2: The outputs $y_{i}$ of the leader and all the followers with topology graph $\overline{\mathscr{G}}$.

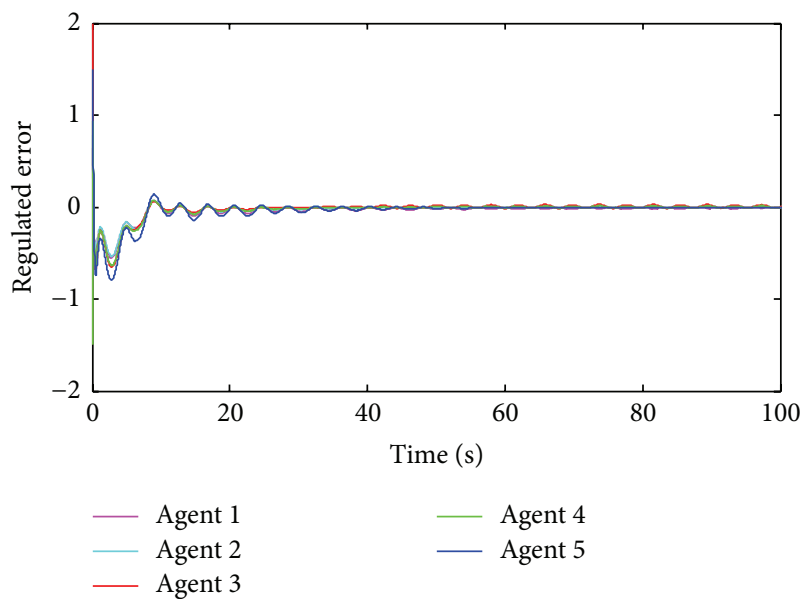

FIGURE 3: The regulated errors $e_{i}(t)$ of all the followers with topology graph $\overline{\mathscr{G}}$.

the followers asymptotically converge to zero. The cooperative output regulation problem of nonlinear multiagent systems under a general directed graph is solved with satisfactory tracking performance of our design.

\section{Summary}

In this paper, the cooperative output regulation problem of nonlinear multiagent systems with a general directed topology graph has been considered. Based on the internal model, distributed feedback controller has been proposed by using the backstepping technique for tracking trajectory and rejecting disturbance. However, the followers in this paper are considered as homogeneous agents while in reality the followers may have different dynamics. In the future work, we have to seek a new controller to cope with the case for heterogeneous multiagent systems in a general directed graph. 


\section{Conflict of Interests}

The authors declare that there is no conflict of interests regarding the publication of this paper.

\section{Acknowledgments}

This work is supported in part by the Research Project of National University of Defense Technology and the National Natural Science Foundation of China (61403406 and 61403410).

\section{References}

[1] X. Wang, T. Liu, and J. Qin, "Second-order consensus with unknown dynamics via cyclic-small-gain method," IET Control Theory \& Applications, vol. 6, no. 18, pp. 2748-2756, 2012.

[2] X. Wang, C. Yu, and Z. Lin, "A dual quaternion solution to attitude and position control for rigid-body coordination," IEEE Transactions on Robotics, vol. 28, no. 5, pp. 1162-1170, 2012.

[3] H. Shi, L. Wang, and T. Chu, "Flocking of multi-agent systems with a dynamic virtual leader," International Journal of Control, vol. 82, no. 1, pp. 43-58, 2009.

[4] Y. Stergiopoulos and A. Tzes, "Spatially distributed area coverage optimisation in mobile robotic networks with arbitrary convex anisotropic patterns," Automatica, vol. 49, no. 1, pp. 232237, 2013.

[5] X. Wang, J. Qin, and C. Yu, "ISS method for coordination control of nonlinear dynamical agents under directed topology," IEEE Transactions on Cybernetics, vol. 44, no. 10, pp. 1832-1845, 2014.

[6] W. Ren, R. W. Beard, and E. M. Atkins, "Information consensus in multivehicle cooperative control," IEEE Control Systems Magazine, vol. 27, no. 2, pp. 71-82, 2007.

[7] W. Zhu and D. Cheng, "Leader-following consensus of secondorder agents with multiple time-varying delays," Automatica, vol. 46, no. 12, pp. 1994-1999, 2010.

[8] L. Gao, X. Zhu, and W. Chen, "Leader-following consensus problem with an accelerated motion leader," International Journal of Control, Automation and Systems, vol. 10, no. 5, pp. 931-939, 2012.

[9] Z. Wang and J. Cao, "Quasi-consensus of second-order leaderfollowing multi-agent systems," IET Control Theory \& Applications, vol. 6, no. 4, pp. 545-551, 2012.

[10] L. Ding, Q.-L. Han, and G. Guo, "Network-based leaderfollowing consensus for distributed multi-agent systems," Automatica, vol. 49, no. 7, pp. 2281-2286, 2013.

[11] Z. Meng, Z. Zhao, and Z. Lin, "On global leader-following consensus of identical linear dynamic systems subject to actuator saturation," Systems and Control Letters, vol. 62, no. 2, pp. 132142, 2013.

[12] Q. Song, J. Cao, and W. Yu, "Second-order leader-following consensus of nonlinear multi-agent systems via pinning control," Systems and Control Letters, vol. 59, no. 9, pp. 553-562, 2010.

[13] H. Li, X. Liao, and G. Chen, "Leader-following finite-time consensus in second-order multi-agent networks with nonlinear dynamics," International Journal of Control, Automation and Systems, vol. 11, no. 2, pp. 422-426, 2013.

[14] Y. Su and J. Huang, "Cooperative output regulation of a linear multi-agent system," in Proceedings of the 30th IASTED
Conference on Modelling, Identification, and Control (AsiaMIC '10), pp. 228-233, Phuket, Thailand, November 2010.

[15] X. Wang, W. Ni, and J. Yang, "Distributed output regulation of switching multi-agent systems subject to input saturation," in Proceedings of the 10th World Congress on Intelligent Control and Automation (WCICA '12), pp. 840-845, IEEE, Beijing, China, July 2012.

[16] S. Li, G. Feng, X. Guan et al., "Distributed adaptive pinning control for cooperative linear output regulation of multi-agent systems," in Proceedings of the 32nd Chinese Control Conference (CCC '13), pp. 6885-6890, Xi'an, China, July 2013.

[17] R. Horn and C. Jornson, Matrix Analysis, Cambridge University Press, New York, NY, USA, 1985.

[18] C. Godsil and G. Royle, Algebraic Graph Theory: Graduate Texts in Mathematics, vol. 207 of Graduate Texts in Mathematics, Springer, New York, NY, USA, 2001.

[19] A. Jadbabaie, J. Lin, and A. S. Morse, "Coordination of groups of mobile autonomous agents using nearest neighbor rules," IEEE Transactions on Automatic Control, vol. 48, no. 6, pp. 988-1001, 2003.

[20] J. Huang, Nonlinear Output Regulation: Theory and Applications, SIAM, Philadelphia, Pa, USA, 2004.

[21] J. Huang and Z. Chen, "A general framework for tackling the output regulation problem," IEEE Transactions on Automatic Control, vol. 49, no. 12, pp. 2203-2218, 2004.

[22] M. A. Barrón and M. Sen, "Synchronization of four coupled van der Pol oscillators," Nonlinear Dynamics, vol. 56, no. 4, pp. 357$367,2009$. 


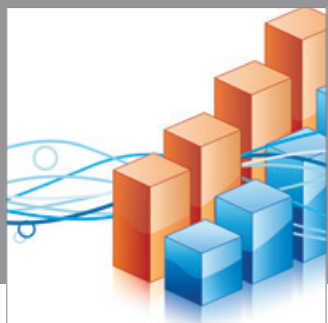

Advances in

Operations Research

mansans

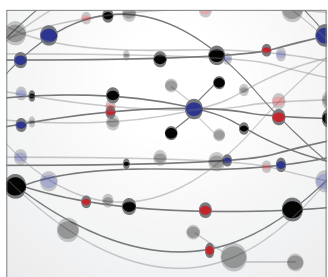

The Scientific World Journal
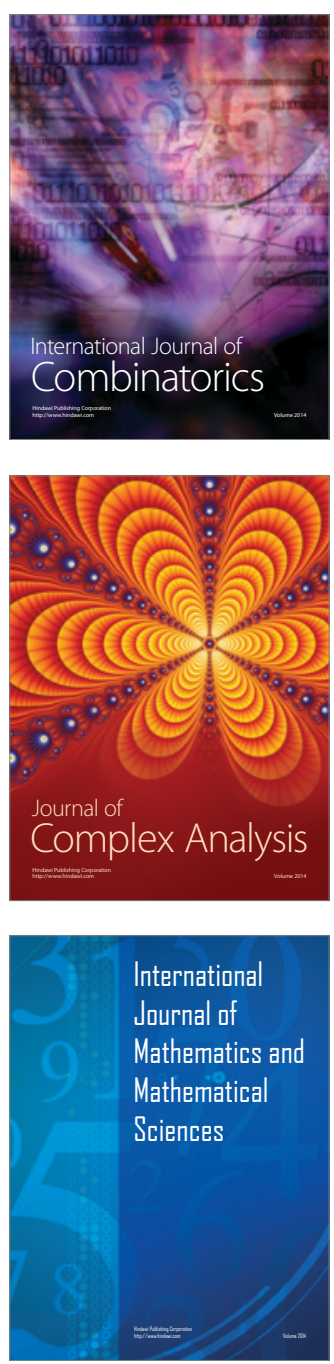
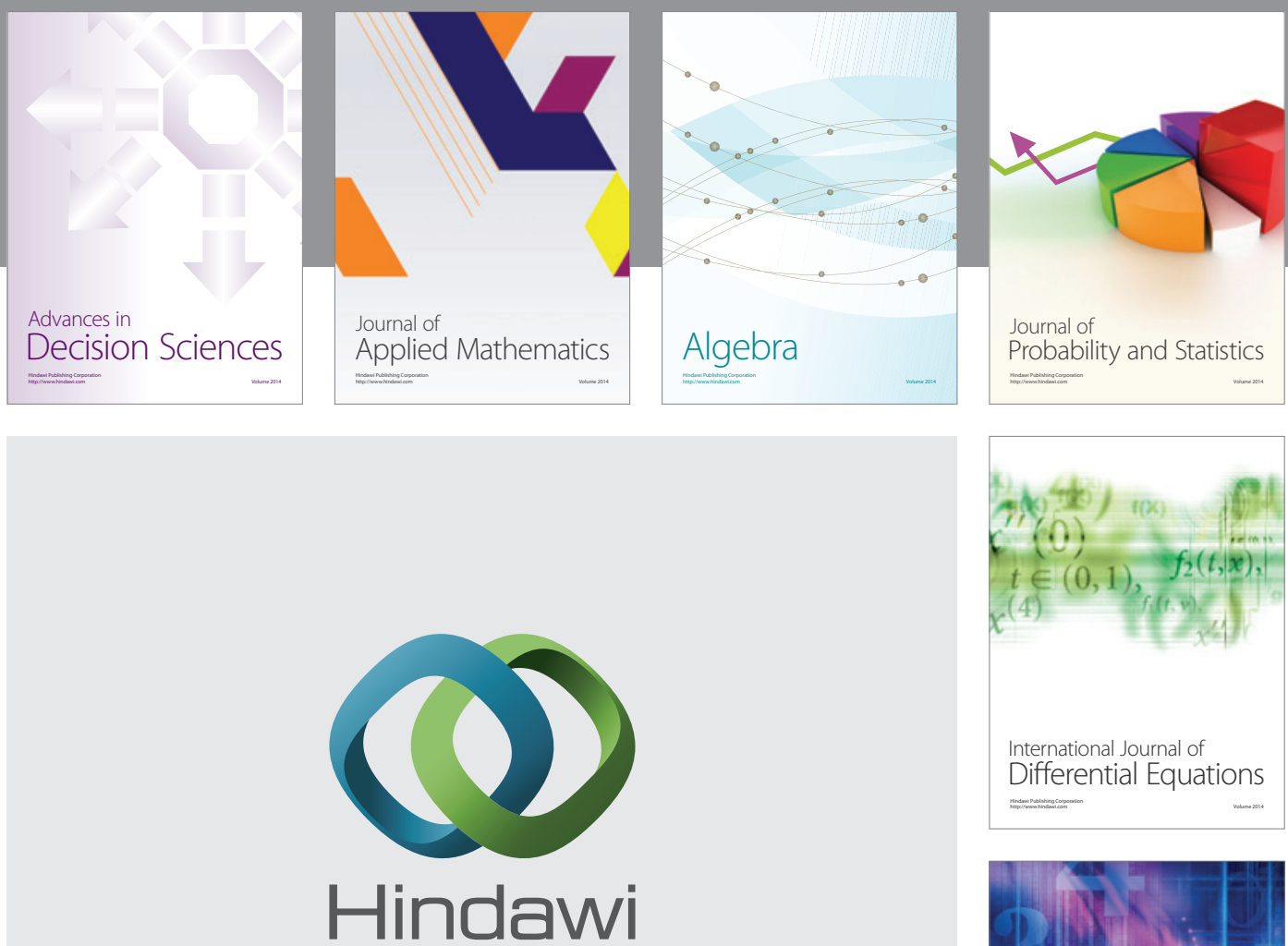

Submit your manuscripts at http://www.hindawi.com
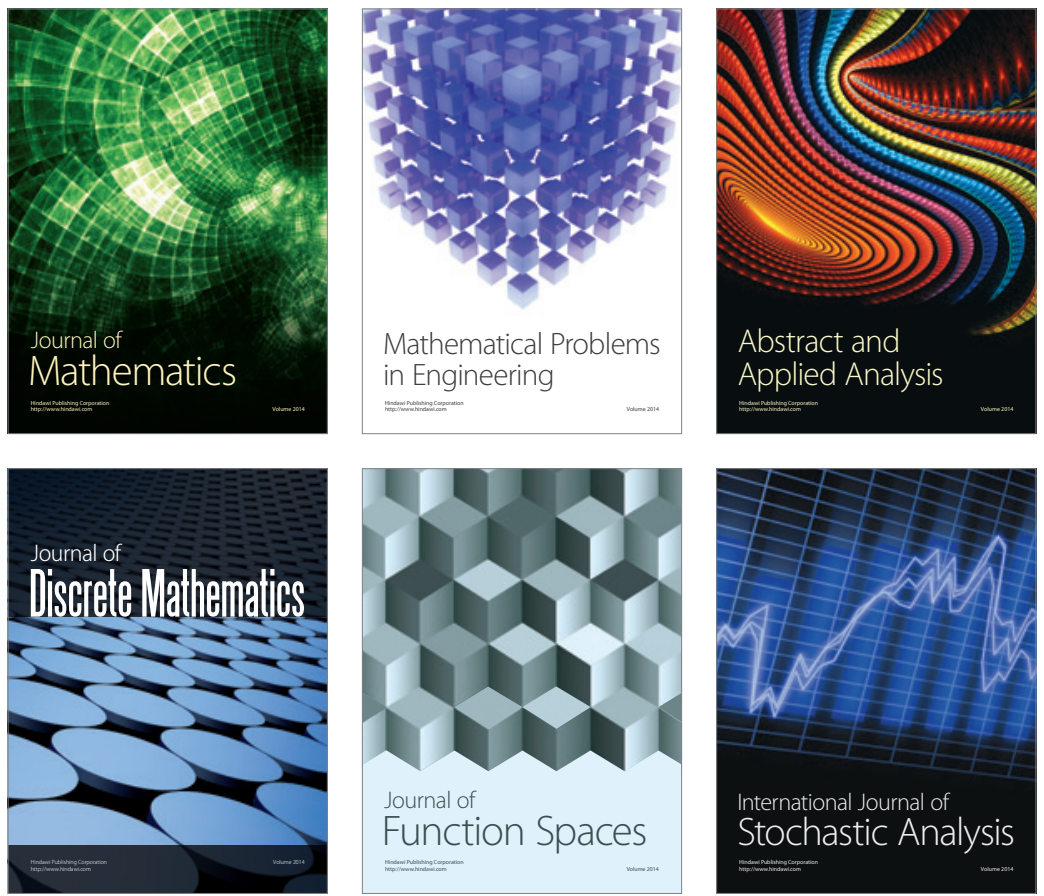

Journal of

Function Spaces

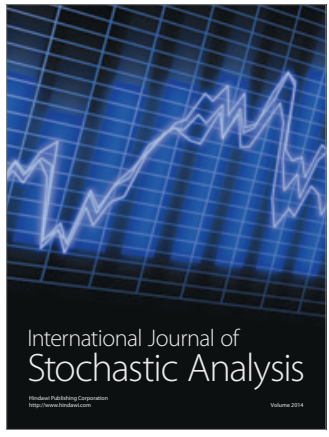

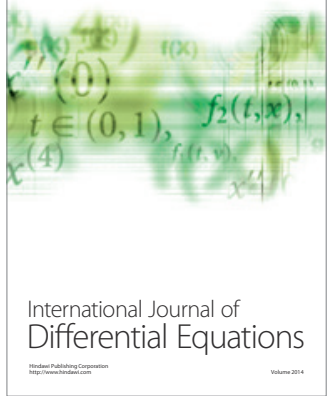
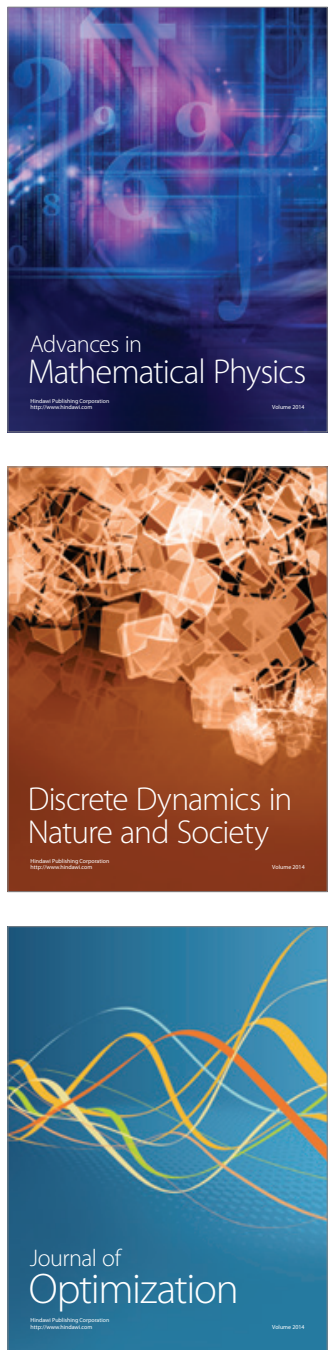